\title{
Do alternative methods for analysing count data produce similar estimates? Implications for meta-analyses
}

\author{
Peter Herbison ${ }^{1 *}$, M. Clare Robertson ${ }^{2}$ and Joanne E. McKenzie ${ }^{3}$
}

\begin{abstract}
Background: Many randomised trials have count outcomes, such as the number of falls or the number of asthma exacerbations. These outcomes have been treated as counts, continuous outcomes or dichotomised and analysed using a variety of analytical methods. This study examines whether different methods of analysis yield estimates of intervention effect that are similar enough to be reasonably pooled in a meta-analysis.

Methods: Data were simulated for 10,000 randomised trials under three different amounts of overdispersion, four different event rates and two effect sizes. Each simulated trial was analysed using nine different methods of analysis: rate ratio, Poisson regression, negative binomial regression, risk ratio from dichotomised data, survival to the first event, two methods of adjusting for multiple survival times, ratio of means and ratio of medians. Individual patient data was gathered from eight fall prevention trials, and similar analyses were undertaken.

Results: All methods produced similar effect sizes when there was no difference between treatments. Results were similar when there was a moderate difference with two exceptions when the event became more common: (1) risk ratios computed from dichotomised count outcomes and hazard ratios from survival analysis of the time to the first event yielded intervention effects that differed from rate ratios estimated from the negative binomial model (reference model) and (2) the precision of the estimates differed depending on the method used, which may affect both the pooled intervention effect and the observed heterogeneity.

The results of the case study of individual data from eight trials evaluating exercise programmes to prevent falls in older people supported the simulation study findings.

Conclusions: Information about the differences in treatments is lost when event rates increase and the outcome is dichotomised or time to the first event is analysed otherwise similar results are obtained. Further research is needed to examine the effect of differing variances from the different methods on the confidence intervals of pooled estimates.
\end{abstract}

Keywords: Count outcomes, Meta-analysis, Methods of analysis, Rates

\section{Background}

Often the outcomes measured in medical research are count outcomes. Typically, these measure the number of times a particular event happens to an individual in a defined period. Examples of count outcomes include the number of falls by the individual, the number of asthma exacerbations or the number of incontinence episodes.

\footnotetext{
* Correspondence: peter.herbison@otago.ac.nz

${ }^{1}$ Department of Preventive and Social Medicine, Dunedin School of Medicine, University of Otago, PO Box 913, Dunedin 9054, New Zealand
} Full list of author information is available at the end of the article
These outcomes are commonly measured in randomised controlled trials (RCTs) to determine the effect of an intervention.

There are many ways of summarising the difference between interventions when the outcome is a count outcome [1-3], such as:

- A simple rate ratio-the ratio of the number of events per person time at risk in each of the treatment groups.

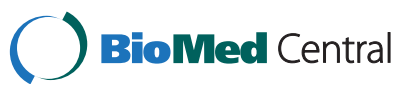

(c) 2015 Herbison et al. Open Access This article is distributed under the terms of the Creative Commons Attribution 4.0 International License (http://creativecommons.org/licenses/by/4.0/), which permits unrestricted use, distribution, and reproduction in any medium, provided you give appropriate credit to the original author(s) and the source, provide a link to the Creative Commons license, and indicate if changes were made. The Creative Commons Public Domain Dedication waiver (http://creativecommons.org/publicdomain/zero/1.0/) applies to the data made available in this article, unless otherwise stated. 
- A rate ratio calculated from the Poisson regression family - such as Poisson and negative binomial.

- A risk ratio after the data are dichotomised into those with and without the event.

- A hazard ratio using the time to the event-either the time to the first event or using a method that copes with multiple times to events.

- A difference in means that treats the data as continuous and is compared using a $t$ test or linear regression. More recently, the ratio of means has been used [4]. These analyses cause few problems for count outcomes with a high mean, such as pulse rate, as the Poisson distribution with a high mean approximates a normal distribution. In practice, however, this approach is often used on data with lower means.

- A difference in medians tested by a non-parametric test such as the Wilcoxon rank sum test or the ratio of medians.

The variety of analytic methods used in RCTs with count outcomes causes difficulties when carrying out a meta-analysis. In addition to the usual problems of heterogeneity arising from populations and treatments, there is heterogeneity in outcomes and analysis methods used across RCTs to evaluate the effect of the intervention. This raises a key question of whether the results from these alternative methods of analysis are comparable enough (exchangeable) to be combined in a meta-analysis.

This paper describes a simulation study designed to see whether mixing the results of different methods of analysis could give reasonable answers in a meta-analysis.

Falling is a major health problem for older people, with approximately $30 \%$ of people over the age of 65 falling each year, with many falls resulting in injury and hospitalisation. The 2009 Cochrane systematic review "Interventions for preventing falls in older people living in the community" included 43 trials that assessed the effect of exercise programmes [5]. The two primary outcomes in this review were the rate of falls and the proportion of fallers. Twenty-six of the 43 studies contributed to the rate of falls meta-analysis, and 31 to the number of fallers. Some studies could not be used because of the way the data were analysed and presented. We asked for individual patient data from randomised trials included in this systematic review, analysed them in different ways and compared the resulting meta-analyses.

\section{Methods}

\section{The simulation study}

Data sets for a two-group parallel RCT with varying parameters were created. The size of each group was randomly chosen from a normal distribution with a mean of 100 and a standard deviation of 2 . This kept the sample sizes of the two arms approximately equal and was large enough to provide stable estimates of the difference between the groups. The number of events experienced for each individual was randomly selected from a Poisson distribution with various means covering the range of potential rates that might be expected in practice. There were two groups of simulations, one with a moderate effect of the intervention, with a rate reduction of approximately $30 \%$ in each of the chosen base values, and one with only a small random difference between groups. There were four groups of simulations ranging from a mean of 0.15 to a mean of 7 for the Poisson distributions when there was no overdispersion. As it is common for count data to have some overdispersion, this was built into the data sets by including 0,20 and $40 \%$ of the numbers of events drawn from Poisson distributions with a higher mean (representing no, moderate and high overdispersion, respectively). Overdispersion was built into both arms of the trial so that the relative differences between the arms would remain approximately the same. As all methods were used on each data set, these minor differences are not problematic. The combinations of parameters for the simulation scenarios with the approximately $30 \%$ reduction in event rate are shown in Table 1 . The simulations with no reduction used the rates, with minor random perturbations, in the control group for both arms.

The treatment period was set at 365 days. About $20 \%$ of observations were randomly chosen to be lost to follow-up after a randomly chosen number of days. The number of days to the events experienced was chosen from a uniform distribution. The simulation started by choosing the sample size and then the number of events in each arm and the follow-up period. Then the time to each of the events was generated. This was followed by the different analyses, and the results were stored on a file. There were 10,000 replications of each simulation scenario. Stata code for one of the simulations is available in the Additional file 1.

Each data set was analysed in nine ways to obtain the following estimates of the intervention effect:

Table 1 Means of the poisson distributions used in the simulations

\begin{tabular}{|c|c|c|c|c|}
\hline & \multicolumn{2}{|c|}{ Base values } & \multicolumn{2}{|c|}{$\begin{array}{l}\text { Overdispersed values (0 \%-no } \\
\text { overdispersion, } 20 \% \text {-moderate } \\
\text { overdispersion and } 40 \% \text {-high } \\
\text { overdispersion from these Median } \\
2.5 \text { and } 97.5 \text { centilesdistributions) }\end{array}$} \\
\hline & Control & Treatment & Control & Treatment \\
\hline Very low & 0.2 & 0.15 & 0.6 & 0.4 \\
\hline Low & 0.5 & 0.35 & 0.8 & 0.6 \\
\hline Medium & 2 & 1.5 & 3 & 2.5 \\
\hline High & 7 & 5 & 10 & 7 \\
\hline
\end{tabular}


1. Simple rate ratio $(\mathrm{RaR})$ calculated from count data (the ratio of the number of events divided by person time of follow-up in the intervention arm to the control arm)

2. Rate ratio estimated from Poisson regression $[2,6]$

3. Rate ratio estimated from the negative binomial regression [7]

4. Risk ratio (RR) calculated from dichotomised data

5. Hazard ratio (HR) estimated from survival analysis for time to the first event [8]

6. Hazard ratio for multiple events estimated using the marginal model [9]

7. Hazard ratio for multiple events estimated using the Andersen-Gill model $[9,10]$

8. The ratio of the mean number of events [4]

9. The ratio of the median number of events.

The negative binomial regressions used the meandispersion model. The marginal model for repeated time to events assumes that individuals are at risk for every event from the time of study entry, whereas the Andersen-Gill method assumes that people are at risk for the second event only from the time they had the first event. People with $n$ events have $n+1$ records in the file, $n$ ending in the event and the $n+1$ th censored at the end of the follow-up. Adjustment is made for multiple records per person in both of these models. See Robertson et al. [11] for more details.

Where possible, information about the $20 \%$ of observations lost to follow-up was included in the analysis [12]. Simple rate ratios were calculated using person days of follow-up, and the Poisson and negative binomial regression models allowed for varying lengths of follow-up through inclusion of an offset in the model. The survival models allow for varying lengths of followup through censoring. However, it is not possible to allow for follow-up time for intervention effect estimates 4,8 and 9 .

Ratios of means and medians (estimates 8 and 9) were used in preference to differences in means and medians, since these ratio measures were comparable with the other estimates of intervention effect although they would not usually be used in practice.

The results of the simulations were examined in several ways. Histograms of the results were produced, with the median, 2.5th and 97.5th centiles of these distributions plotted using forest plots. In addition, to examine the exchangeability of the methods, we compared the estimated intervention effect from each method of analysis with the estimated rate ratio from the negative binomial regression using two metrics. The first metric compared the mean of the differences in the two estimates (for ease of interpretation) across the 10,000 replicates, while the second metric compared the mean of the ratios of the two estimates (since the underlying scale is relative). The rate ratio from the negative binomial regression model was chosen as the reference estimate. The negative binomial model is a more general model compared with the Poisson regression model that relaxes the strong assumption that the underlying rate of the outcome is the same for each included participant. The negative binomial model requires the additional estimation of a dispersion parameter (which will make it less efficient than the Poisson model in the absence of overdispersion); however, the model is theoretically more plausible $[11,13,14]$. The mean and standard deviation of the differences in the estimates are presented in the "Results" section and of the ratios in the Additional file 1. The interpretation of a positive mean difference is that the estimate for the comparison method was closer to $1 \mathrm{com}-$ pared with the estimate from the negative binomial regression. Mean differences enable a judgement about whether there is, on average, an important difference between the estimates calculated from each of the analytical methods and the comparison method. The standard deviation of the differences gives an indication of how close the average result is to the negative binomial rate ratio, with large values indicating that the estimates are not always close and that for any particular trial, the use of an alternative method may result in an importantly different estimate of intervention effect.

Stata V13 (StataCorp, College Station, TX, USA) was used for all simulations.

\section{The case study}

All of the corresponding authors of the trials that contributed to the comparison of multiple component group or home-based exercise programmes versus no exercise programme in the 2009 Cochrane systematic review were invited by e-mail to contribute to this part of the study.

The e-mail provided authors with information and preliminary results of the simulation study and informed them of the purpose of this empirical study. Authors were then asked whether they would consider taking part in the empirical study. Those opting to participate in the empirical study were sent a second e-mail and given the option of undertaking a series of analyses themselves (and contributing the results) or providing de-identified individual participant data sets, which we would analyse. All authors who agreed to take part chose the latter option.

Each data set was analysed to estimate the effect of exercise versus no exercise using a (1) simple RaR, (2) RR calculated from the dichotomised outcome (fallers and non-fallers), (3) RaR estimated from Poisson regression, (4) RaR estimated from the negative binomial regression and (5) the ratio of means. The median number of falls in all groups was zero, so the ratio of medians could not be computed. Nor was it possible to undertake survival 
analyses because most studies either did not collect the times of the falls or did not provide this data. One trial was cluster randomised and so the Poisson regression and negative binomial regression were allowed for the potential within-cluster correlation [15].

For each analytical method, estimates of the intervention effect were pooled using both fixed and random effects meta-analytic models using the metan routine in Stata [16]. Fixed effect meta-analyses used the method of Mantel and Haenszel [17], and the random effects models used the method of DerSimonian and Laird [18].

\section{Results}

The following are results for the simulations with the approximately $30 \%$ reduction in treatment effect.

\section{Simulations with a very low mean}

Simulations with a very small mean and no overdispersion yielded estimates for all analytical methods that were similar to the negative binomial rate ratio (Fig. 1, Table 2). The percentile-based confidence intervals (CIs) around the estimates are very similar for all the methods (Fig. 1). The full distributions of the results are given in Additional file 1: Figures 1a to 1d. Dichotomising the data into event/no event yielded the largest difference, with an average RR of 0.8088 compared with 0.7941 from the negative binomial regression (an increase of $3 \%$ (Additional file 1: Table S1)), but this is unlikely to change the interpretation of the result. The $95 \%$ CIs become narrower as overdispersion increased but this was less so for the three survival analysis methods (Fig. 1). While the estimates from the other methods seem to be little affected by overdispersion, dichotomising the outcome yields a RR closer to 1 by 0.0481 for high overdispersion (an increase of $8 \%$ ), which may impact on clinical interpretation. The HR for time to the first event is the second most different result (0.7998 vs negative binomial $0.7941,1 \%$ increase), and this increases slightly with overdispersion (to a $4 \%$ increase). Unsurprisingly, the estimates from the Poisson regression and the simple $\mathrm{RaR}$ are always exactly the same. Dichotomising the data, and the three survival analysis methods, have the largest standard deviations, suggesting that for any particular data set, the estimates computed by these analytical methods may differ substantially from the negative binomial RaR. The ratio of medians is impossible to calculate for low means as the median is zero if fewer than $50 \%$ of participants in either group do not have the event.

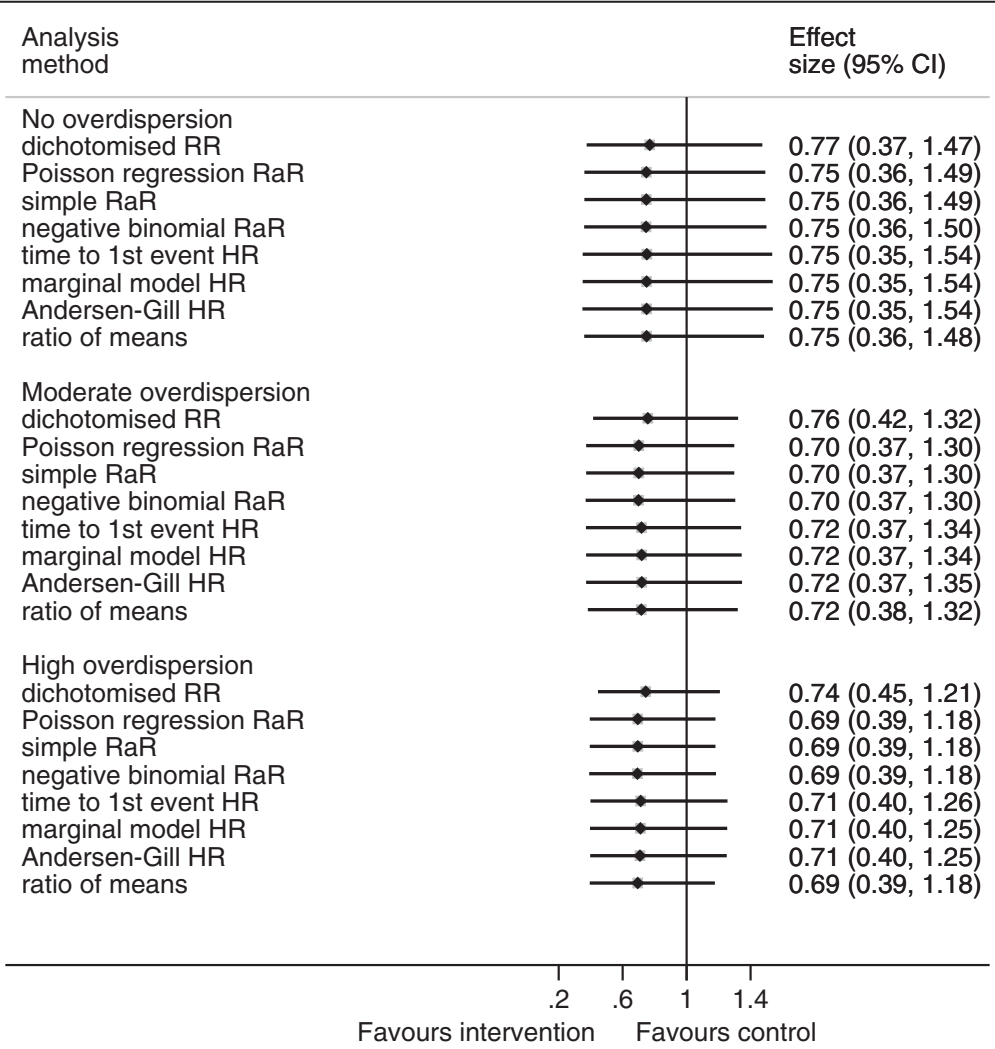

Fig. 1 Median, 2.5 and 97.5 centiles (percentage-based confidence intervals) from the simulation results for the very low mean (control 0.2, treatment 0.15 ) 
Table 2 Simulation results for the very low mean (control 0.2, treatment 0.15)

\begin{tabular}{|c|c|c|c|c|c|c|}
\hline & No overdispersion & & Moderate overdis & & High overdispers & \\
\hline & $\operatorname{RaR}^{\mathrm{a}}=0.79$ & & $\operatorname{RaR}^{\mathrm{a}}=0.73$ & & $\operatorname{RaR}^{\mathrm{a}}=0.72$ & \\
\hline & Mean difference ${ }^{b}$ & SD & Mean difference & SD & Mean difference & SD \\
\hline Dichotomise RR & 0.01 & 0.09 & 0.05 & 0.09 & 0.05 & 0.09 \\
\hline Poisson RaR & $<0.01$ & 0.01 & $<0.01$ & 0.01 & $<0.01$ & 0.01 \\
\hline Simple RaR & $<0.01$ & 0.01 & $<0.01$ & 0.01 & $<0.01$ & 0.01 \\
\hline Time to the first event HR & 0.01 & 0.09 & 0.02 & 0.10 & 0.02 & 0.09 \\
\hline Marginal model HR & 0.01 & 0.09 & 0.02 & 0.09 & 0.02 & 0.09 \\
\hline Andersen-Gill HR & $<0.01$ & 0.09 & 0.02 & 0.09 & 0.02 & 0.09 \\
\hline Ratio of means & $<0.01$ & 0.03 & 0.02 & 0.03 & $<0.01$ & 0.03 \\
\hline Ratio of medians & Not possible as bc & ians ar & & & & \\
\hline
\end{tabular}

SD standard deviation

${ }^{\text {a }}$ Rate ratio from negative binomial regression model

${ }^{\mathrm{b}}$ Mean of the estimate minus negative binomial rate ratio

\section{Simulation scenarios with a low mean}

With a slightly larger mean, the intervention effect estimates based on the dichotomised outcomes were, on average, importantly closer to the null compared with those estimated from the negative binomial regression model (Fig. 2, Table 3). The width of the percentilebased CIs of the estimated intervention effects were similar across the methods, although the width for the dichotomised outcome was slightly narrower (Fig. 1). The dichotomised RR was 8-9 \% larger than the negative binomial RaR (Additional file 1: Table S2). Results for the other methods are similar to those using the lower mean. The histograms for these results are in Additional file 1: Figures $2 \mathrm{a}-\mathrm{d}$.

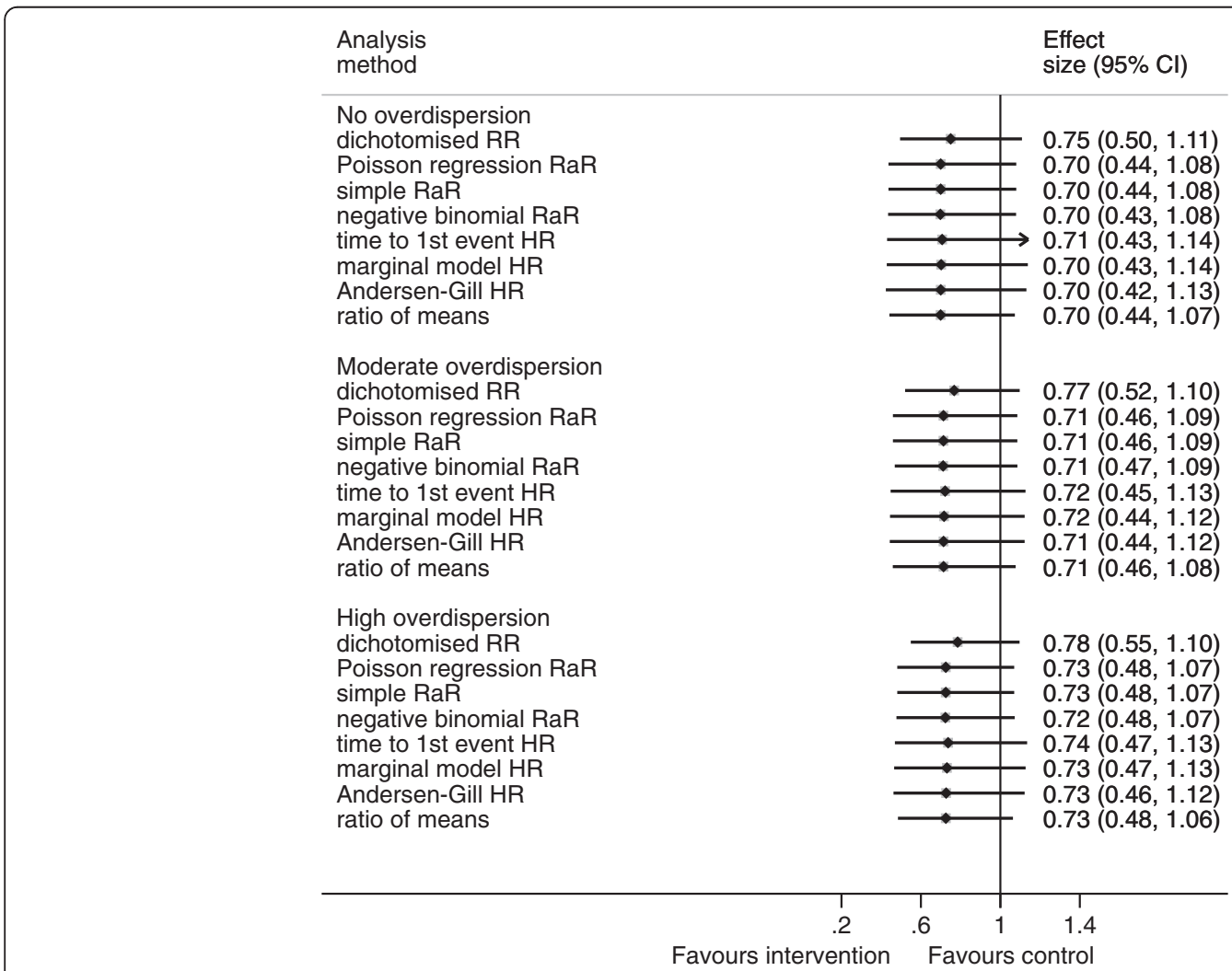

Fig. 2 Median, 2.5 and 97.5 centiles (percentage-based confidence intervals) from the simulation results for the low mean (control 0.5, treatment 0.35) 
Table 3 Simulation results for the low mean (control 0.5, treatment 0.35)

\begin{tabular}{|c|c|c|c|c|c|c|}
\hline & \multicolumn{2}{|l|}{ No overdispersion } & \multicolumn{2}{|c|}{ Moderate overdispersion } & \multicolumn{2}{|c|}{ High overdispersion } \\
\hline & \multicolumn{2}{|l|}{$\operatorname{RaR}^{a}=0.71$} & \multicolumn{2}{|l|}{$\operatorname{RaR}^{\mathrm{a}}=0.73$} & \multicolumn{2}{|l|}{$\operatorname{RaR}^{a}=0.74$} \\
\hline & Mean difference $^{b}$ & SD & Mean difference & SD & Mean difference & SD \\
\hline Dichotomise RR & 0.05 & 0.08 & 0.05 & 0.08 & 0.06 & 0.08 \\
\hline Poisson RaR & $<0.01$ & 0.06 & $<0.01$ & 0.01 & $<0.01$ & 0.01 \\
\hline Simple RaR & $<0.01$ & 0.06 & $<0.01$ & 0.01 & $<0.01$ & 0.01 \\
\hline Time to the first event $\mathrm{HR}$ & 0.01 & 0.08 & 0.01 & 0.09 & 0.02 & 0.09 \\
\hline Marginal model HR & 0.01 & 0.08 & 0.01 & 0.08 & 0.01 & 0.08 \\
\hline Andersen-Gill HR & 0.01 & 0.08 & 0.01 & 0.08 & 0.01 & 0.08 \\
\hline Ratio of means & $<0.01$ & 0.03 & $<0.01$ & 0.03 & $<0.01$ & 0.03 \\
\hline Ratio of medians & \multicolumn{6}{|c|}{ Only possible for 292, 717 and 2087 of the 10,000 simulations, respectively } \\
\hline
\end{tabular}

$S D$ standard deviation

${ }^{\text {a }}$ Rate ratio from negative binomial regression model

${ }^{\mathrm{b}}$ Mean of the estimate minus negative binomial rate ratio

\section{Simulation scenarios with a moderate mean}

When the mean in the control group is 2, dichotomising the data produces an estimate of effect much closer to 1 than the negative binomial RaR (Fig. 3, Table 4). The percentile-based confidence interval around the dichotomised RR is narrower than the other CIs (Fig. 3). The HR estimates from time to the first event analyses also move closer to 1 , and the differences between the $\mathrm{HR}$ estimates and the RaR are more variable. Adjusting for multiple survival times, by using the marginal or Andersen-Gill method, seems to lessen the difference, but the differences have relatively large standard deviations, so individual intervention effects may be quite discrepant. The Andersen-Gill method yields estimates that are slightly further from 1 than the negative binomial regression estimates, but usually not enough to influence interpretation. The ratio of medians produces an average estimate closer to 1 than the negative binomial RR. The distribution of the ratio of medians is highly concentrated at discrete values (Additional file 1: Figure S3a, b, and c). The differences have the largest standard deviation across all levels of overdispersion, so that results from individual studies using this method may differ importantly from the negative binomial estimate.

\section{Simulation scenarios with a large mean}

With a mean in the control of 7 , dichotomising these data sets yielded, on average, RRs close to 1 (Fig. 4, Table 5). The percentile-based CI is very narrow (Fig. 4). The time to the first event analysis was also suggestive of no intervention effect. The confidence intervals around the negative binomial RaR are wider than those around the simple RaR and the Poisson RaR. The percentile-based confidence intervals for the three survival analyses were wider than the other confidence intervals (Fig. 4). Adjusting for multiple survival times gave estimates closer to the negative binomial RaR, with the marginal model closer than Andersen-Gill. The results for the Andersen-Gill method are even further from one than the negative binomial $\mathrm{RaR}$ than with a smaller mean, yielding estimates that are importantly different between the two analytical methods. The standard deviations for the differences between the HR and the estimate from the negative binomial model in both of the marginal and Andersen-Gill methods were large. The ratio of medians yields similar estimates, on average, compared with the negative binomial $\mathrm{RaR}$ in scenarios with large means with confidence limits similar to those from the negative binomial regression (Fig. 4). This is despite the distribution of the ratios of medians being very non-normal.

\section{Convergence}

The negative binomial regression did not always converge and in these instances yielded the same intervention estimates as the Poisson regression (Table 6). Non-convergence was greatest in simulation scenarios where there was no overdispersion and was therefore unlikely to affect the results of the simulations.

The ratio of medians was impossible to calculate for the very low mean, and for the low mean, the ratio of medians was only possible for 292, 717 and 2087 of the 10,000 simulations for no, moderate and high overdispersion, respectively.

\section{The empirical case study}

Eight (of the possible 43) data sets were provided, each containing (by participant) the number of falls during the trial, group allocation (exercise, no exercise), and the time period of falls were monitored $[15,19-25]$. Some details of these eight studies are presented in Table 7.

Meta-analysing risk ratios from dichotomised outcomes yielded estimates of intervention effect that 


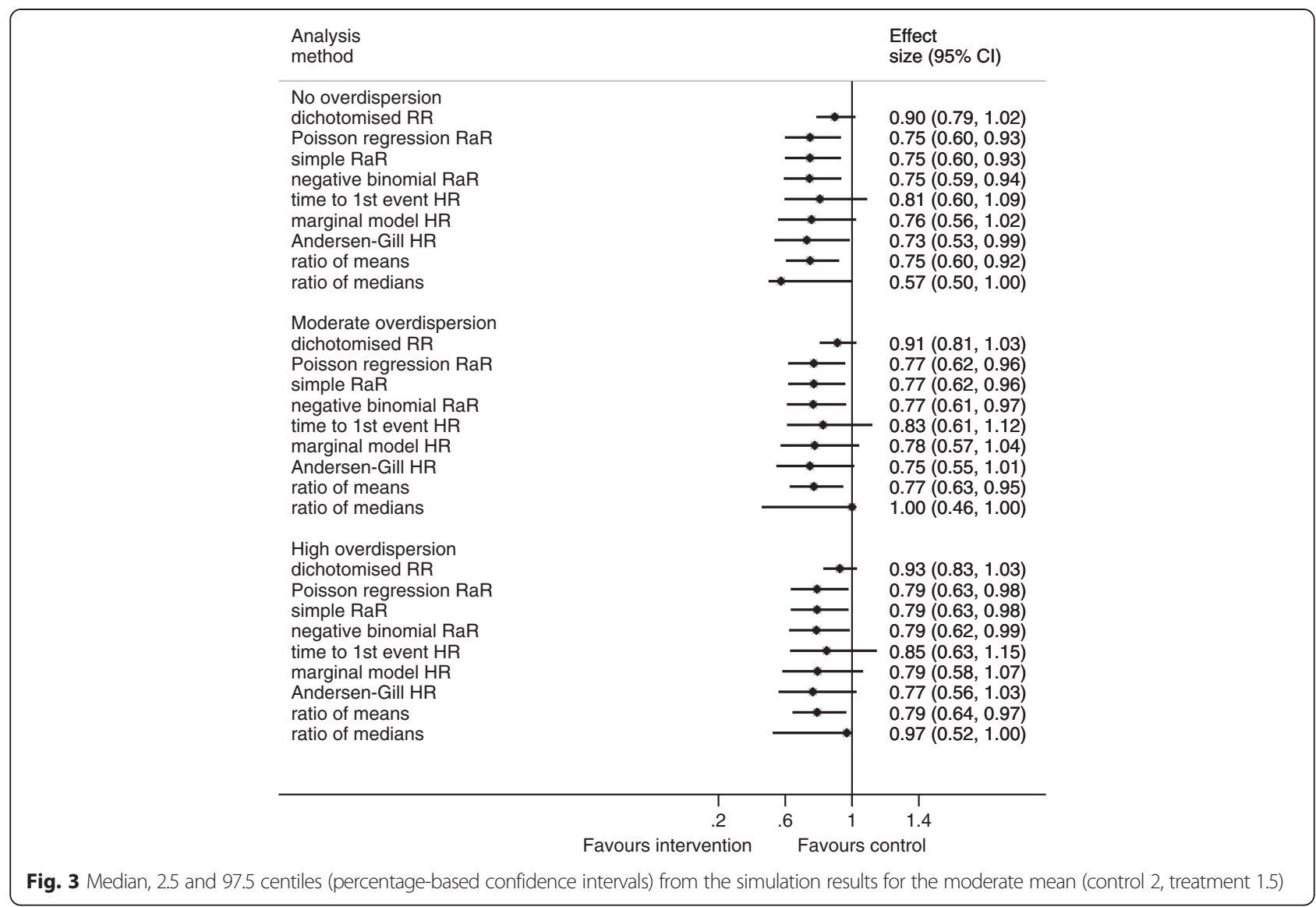

differed from those from the other analytical methods (Fig. 5). The other methods all provided very similar results. There was little variation in the precision of the fixed effect estimate across the analytical methods. However, the $95 \% \mathrm{CI}$ of the random effects estimate based on the dichotomised outcomes was notably narrower than the $95 \%$ CI for other random effects analyses.

\section{No difference between groups}

When there was no difference in the effect of treatment in the groups, all methods gave very similar results for all scenarios.

\section{Discussion}

The results of this study suggest that it may well be possible in many situations to combine in a meta-analysis

Table 4 Simulation results for the moderate mean (control 2, treatment 1.5)

\begin{tabular}{|c|c|c|c|c|c|c|}
\hline & \multicolumn{2}{|l|}{ No over dispersion } & \multicolumn{2}{|c|}{ Moderate overdispersion } & \multicolumn{2}{|c|}{ High overdispersion } \\
\hline & \multicolumn{2}{|l|}{$\operatorname{RaR}^{a}=0.75$} & \multicolumn{2}{|c|}{$\operatorname{RaR}^{a}=0.77$} & \multicolumn{2}{|c|}{$\operatorname{RaR}^{a}=0.79$} \\
\hline & Mean difference ${ }^{b}$ & SD & Mean difference & SD & Mean difference & SD \\
\hline Dichotomise RR & 0.15 & 0.07 & 0.14 & 0.08 & 0.14 & 0.08 \\
\hline Poisson RaR & $<0.01$ & 0.01 & $<0.01$ & 0.01 & $<0.01$ & 0.01 \\
\hline Simple RaR & $<0.01$ & 0.01 & $<0.01$ & 0.01 & $<0.01$ & 0.01 \\
\hline Time to the first event HR & 0.07 & 0.11 & 0.06 & 0.11 & 0.07 & 0.12 \\
\hline Marginal model HR & 0.02 & 0.09 & 0.01 & 0.09 & 0.01 & 0.10 \\
\hline Andersen-Gill HR & -0.01 & 0.08 & -0.02 & 0.09 & -0.02 & 0.09 \\
\hline Ratio of means & $<0.01$ & 0.03 & $<0.01$ & 0.04 & $<0.01$ & 0.04 \\
\hline Ratio of medians & 0.05 & 0.19 & 0.09 & 0.17 & 0.07 & 0.14 \\
\hline
\end{tabular}

SD standard deviation

${ }^{\mathrm{a}}$ Rate ratio from negative binomial regression model

${ }^{\mathrm{b}}$ Mean of the estimate minus negative binomial rate ratio 


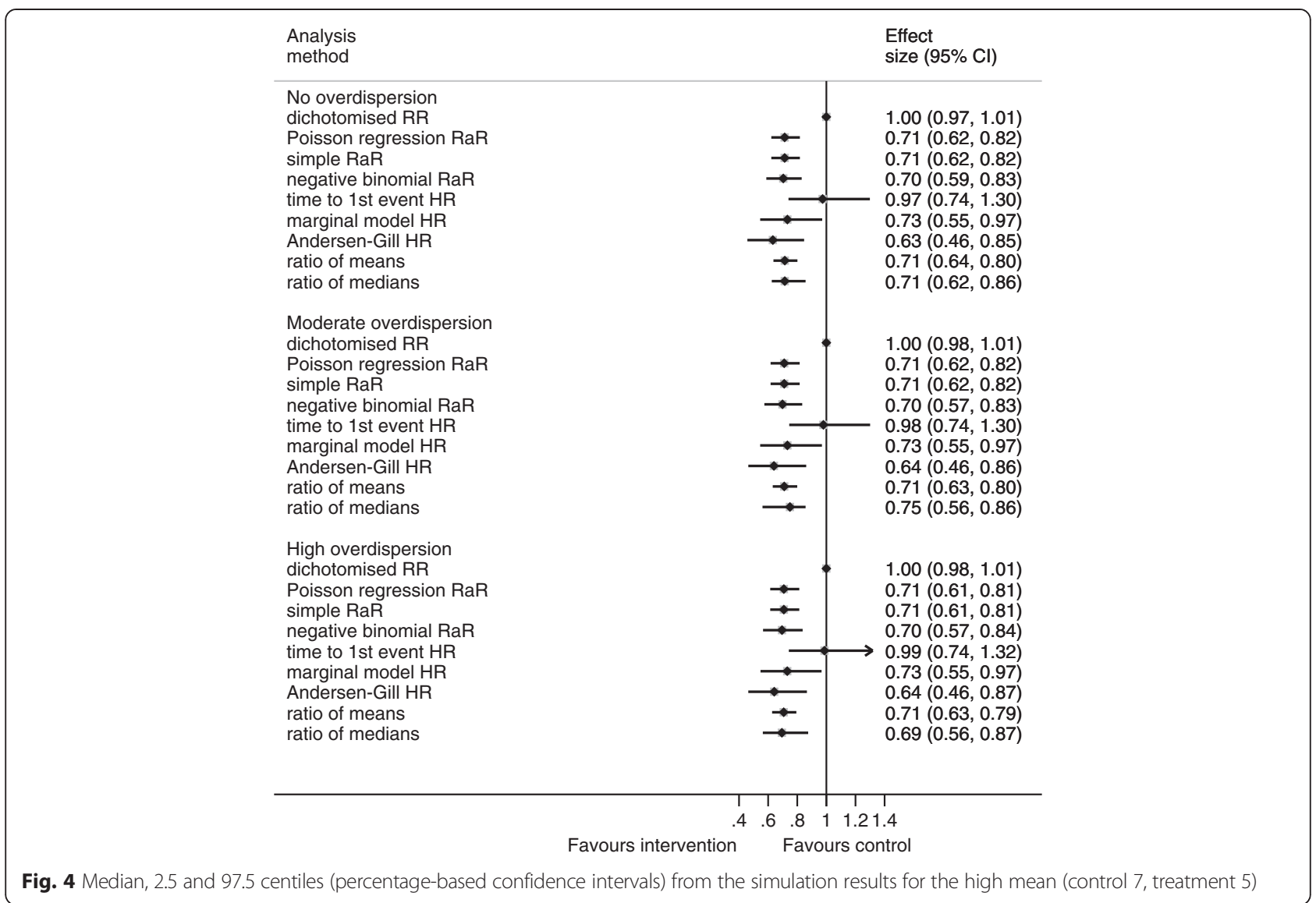

the estimates of intervention effects for count outcomes analysed in various ways, as the results from the different analysis methods were very similar. Apart from a few instances, most analyses gave estimates that were on average close to the $\mathrm{RaR}$ from a negative binomial regression. Further, examination of the range of data from the simulations showed that the confidence intervals of most of the methods were similar. Therefore, pooling intervention estimates calculated by different methods is likely to be generally reasonable. This has been shown using both simulations and actual data from a metaanalysis of RCTs. When events were rare, or there was no treatment effect, all methods of analysis provide a very similar estimate of intervention effect with similar variation. An exception to this is the ratio of medians, which is impossible to calculate unless both groups have

Table 5 Simulation results for the high mean (control 7, treatment 5)

\begin{tabular}{|c|c|c|c|c|c|c|}
\hline & \multicolumn{2}{|l|}{ No overdispersion } & \multicolumn{2}{|c|}{ Moderate overdispersion } & \multicolumn{2}{|c|}{ High overdispersion } \\
\hline & \multicolumn{2}{|l|}{$\operatorname{RaR}^{\mathrm{a}}=0.71$} & \multicolumn{2}{|c|}{$\operatorname{RaR}^{a}=0.70$} & \multicolumn{2}{|l|}{$\operatorname{RaR}^{a}=0.70$} \\
\hline & Mean difference ${ }^{b}$ & SD & Mean difference & SD & Mean difference & SD \\
\hline Dichotomise RR & 0.29 & 0.06 & 0.30 & 0.07 & 0.30 & 0.07 \\
\hline Poisson RaR & 0.01 & 0.02 & 0.01 & 0.03 & 0.01 & 0.03 \\
\hline Simple RaR & 0.01 & 0.02 & 0.01 & 0.03 & 0.01 & 0.03 \\
\hline Time to the first event HR & 0.28 & 0.14 & 0.29 & 0.14 & 0.30 & 0.15 \\
\hline Marginal model HR & 0.03 & 0.10 & 0.04 & 0.10 & 0.04 & 0.10 \\
\hline Andersen-Gill HR & -0.07 & 0.09 & -0.05 & 0.10 & -0.05 & 0.10 \\
\hline Ratio of means & 0.01 & 0.04 & 0.01 & 0.05 & 0.01 & 0.05 \\
\hline Ratio of medians & 0.01 & 0.06 & 0.02 & 0.07 & 0.01 & 0.06 \\
\hline
\end{tabular}

SD standard deviation

${ }^{\mathrm{a}}$ Rate ratio from negative binomial regression model

${ }^{\mathrm{b}}$ Mean of the estimate minus negative binomial rate ratio 
Table 6 Rates of non-convergence for negative binomial regression from the 10,000 simulations

\begin{tabular}{lllll}
\hline & Very low mean (\%) & Low mean (\%) & Moderate mean (\%) & High mean (\%) \\
\hline No overdispersion & 8.15 & 6.99 & 1.37 & 0.04 \\
Moderate overdispersion & 3.77 & 5.28 & 0.33 & 0 \\
High overdispersion & 2.62 & 4.29 & 0.20 & 0 \\
\hline
\end{tabular}

more than $50 \%$ of participants with events. As events become more common, dichotomising the results into those with the event and those without increasingly loses the ability to discriminate between treatments, and the confidence interval becomes narrower. Intuitively, as events become more common, it is likely that all, or almost all, of the participants will experience one or more events. Similarly, time to the first event loses the ability to discriminate with increasing event rates, but this happens more slowly than with dichotomising the data.

Poisson regression and negative binomial regression models gave very similar results for the RaR, even when there was a significant amount of overdispersion. This was expected given these distributions have the same expected value $[13,26]$. The standard error of the RaR estimated from Poisson regression will be too small in the presence of overdispersion, which will have implications for the weights in meta-analytic models. In this simulation, the underestimation of the standard error was only slight but was most noticeable with both a high mean and a lot of overdispersion. Trials that are analysed using Poisson regression in the presence of overdispersion will receive too much weight in the meta-analysis. The impact of not allowing for overdispersion, and subsequent underestimation of the variance of the intervention effect, was evident when comparing the fixed effect meta-analysis confidence intervals calculated from using Poisson regression compared with the negative binomial regression in the empirical study.

Adjusting the survival analyses for multiple events also gave estimates close to those from the negative binomial regression, although the confidence intervals were wider, especially as the mean increases. An exception to this was the Andersen-Gill method that gave an estimate of the HR that was, on average, slightly further from 1 than the negative binomial RaR. The difference between the estimates increases as the mean increases, which may lead to a different interpretation of the intervention effect and make it unreasonable to combine Andersen-Gill HR estimates with those estimated from the negative binomial regression. All survival models in these simulations make the assumption of proportional hazards. In our simulations, the proportional hazards assumption is likely to be true because of the way the data was generated but may not be so for any particular RCT.

The ratio of medians is clearly inappropriate where the event rate is low as the medians in one or both groups are likely to be zero. As the event rate increases, the average difference between estimates calculated from the

Table $\mathbf{7}$ Characteristics of studies in the empirical study

\begin{tabular}{|c|c|c|c|c|}
\hline Study name & Follow-up & Interventions & Number in arm & Number of falls \\
\hline \multirow[t]{2}{*}{ Barnett et al. 2003 [19] } & \multirow[t]{2}{*}{12 months } & Group exercise with home exercise plan & 76 & 40 \\
\hline & & No exercise & 74 & 70 \\
\hline \multirow[t]{2}{*}{ Campbell et al. 1997 [20] } & \multirow[t]{2}{*}{24 months } & Individualised supervised home exercise & 116 & 88 \\
\hline & & No treatment & 117 & 152 \\
\hline \multirow[t]{2}{*}{ Campbell et. al 1999 [21] } & \multirow[t]{2}{*}{10 months } & Individualised supervised home exercise & 45 & 22 \\
\hline & & No treatment & 48 & 35 \\
\hline \multirow[t]{2}{*}{ Green et al. 2002 [22] } & \multirow[t]{2}{*}{9 months } & Individualised physiotherapy exercise in the community & 85 & 74 \\
\hline & & No treatment & 85 & 51 \\
\hline \multirow[t]{2}{*}{ Lord et al. 1995 [23] } & \multirow[t]{2}{*}{12 months } & Exercise classes & 75 & 44 \\
\hline & & No treatment & 94 & 75 \\
\hline \multirow[t]{2}{*}{ Lord et al. 2003 [19] } & \multirow[t]{2}{*}{12 months } & Group exercise & 259 & 174 \\
\hline & & No treatment or relaxation exercises & 249 & 211 \\
\hline \multirow[t]{2}{*}{ Robertson et al. 2001 [24] } & \multirow[t]{2}{*}{12 months } & Individualised supervised home exercise & 121 & 80 \\
\hline & & No exercise & 119 & 109 \\
\hline \multirow[t]{2}{*}{ Skelton et al. 2005 [25] } & \multirow[t]{2}{*}{36 weeks } & Individualised supervised group and home exercise & 50 & 66 \\
\hline & & Pamphlet on home exercises & 31 & 119 \\
\hline
\end{tabular}




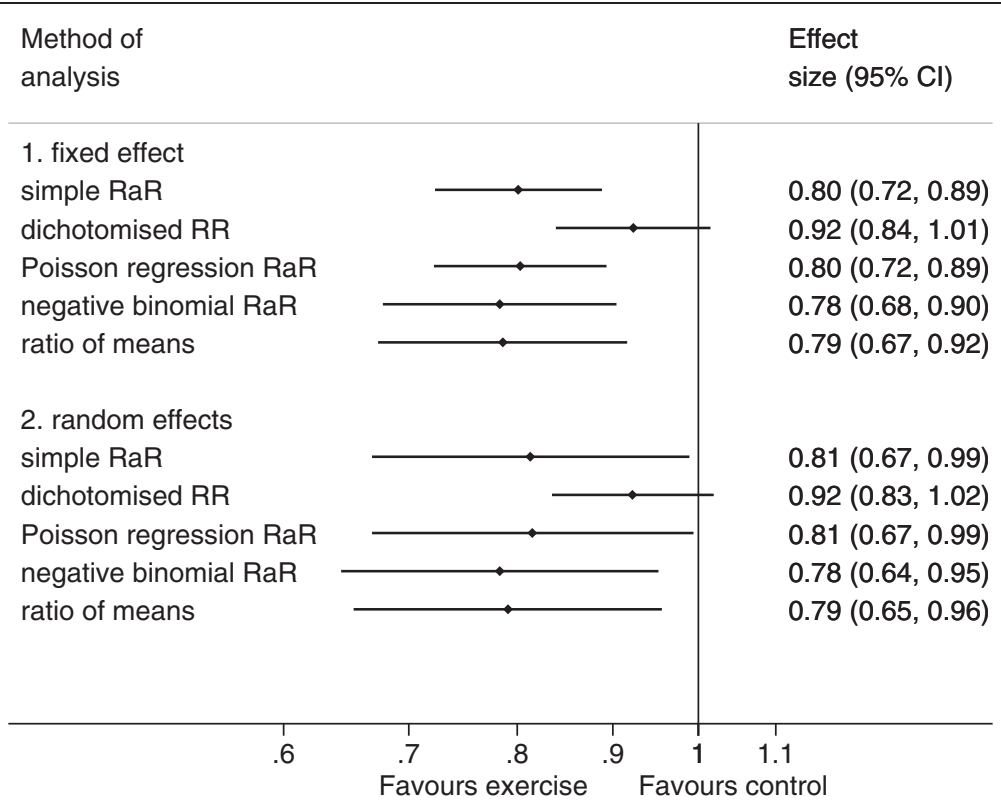

Fig. 5 Pooled estimates of the effect of exercise in the prevention of falls using different methods of analysis calculated using individual patient data from eight studies

ratio of medians and negative binomial regression is small. However, in any particular trial, the difference could be large, as indicated by the large standard deviation of the differences. Especially when the mean is low, the distribution of the ratio of medians is highly concentrated at discrete values but becomes smoother as the mean increases. This could lead to different variances compared with the other models. In practice, it is difficult to use the ratio of medians as the standard error cannot be computed from commonly reported statistics. There is a formula for the $95 \%$ confidence interval of the ratio of medians, but calculation requires the original data [27]. An alternative to using this formula, but still requiring the original data, is to use a method such as bootstrapping to compute the standard errors. More commonly, trial authors will report one of the other effect measures, such as the simple RaR (or at least the raw data that allows this ratio to be calculated). Calculation of the ratio of means is likely to be possible from many studies where the means are reported. There is a standard formula that calculates an approximate standard error from the mean, standard deviation and number of individuals in each of the arms of the study [4].

It is perhaps unsurprising that the estimates and their distributions are similar. The simple RaR and Poisson regression estimate the same parameter; any differences are likely to be due to rounding errors, as the Poisson regression requires more calculations to be performed. The expected values of the estimates from Poisson regression and negative binomial regression are the same. Survival analysis and Poisson regression estimate the same parameter when the baseline hazard is constant [28], which in these simulations will hold, and should for many RCTs. The ratio of means is the coefficient from a linear regression of group assignment on the log of the count outcomes. This is similar to the coefficient in a Poisson regression, except that linear regression does not cope well with zero scores in the outcome, the error structure is different and it is unable to adjust for different follow-up periods.

We chose the negative binomial model as the reference model as it seems appropriate for this sort of data, especially in the presence of overdispersion. This does not allow for the estimation of bias in any of the methods, as we do not have the "true" value. As the question we wanted to answer was whether the results of the different methods could be combined in a meta-analysis looking at the difference from one of the methods was more appropriate.

There are other possibilities for the analysis of count outcomes, such as zero inflated Poisson, zero inflated negative binomial and Poisson regression with robust errors which allows for overdispersion by relaxing the requirement that the mean and variance are equal. However, we did not evaluate these methods since they are not used very often in practice.

Previously, it has been established that, to prevent bias, it is important to account for the length of exposure, which may differ because of dropouts that are not missing at random [12, 29]. The simple rate ratio, Poisson regression and negative binomial regression are all able to adjust for varying follow-up times, as do the survival analysis methods. Thus, it is surprising that the ratio of 
means and the ratio of medians yield similar effect estimates to those estimated from the negative binomial regression. This may be a result of the data sets generated assuming similar attrition across groups, and the missing data mechanism being participants missing completely at random. Under various scenarios (e.g. varying attrition rates and different missing data mechanisms (e.g. not missing at random)), the ratio of means and ratio of medians may yield effect estimates that differ compared with those estimated from negative binomial regression.

The choice of a uniform distribution to pick the times that the events occurred may not be the most realistic option. Events may be more likely to occur closer together or further apart than a uniform distribution would give. They also may not be independent of each other, particularly as having an event may increase or decrease the time to the next event and this may depend on the nature of the event.

The fact that intervention effect estimates from RCTs using different analytical methods can, in some circumstances, be pooled in a meta-analysis should not make the method of analysis a random choice in any particular trial. The analysis should match the hypothesis and the study design. We have previously advocated for the use of negative binomial regression in evaluating falls prevention studies [11], as have others for this type of data [14]. Negative binomial regression allows for all events to be included (thus using all information) and the length of exposure to vary and more appropriately accounts for overdispersed data. But it does treat individuals who have multiple events in quick succession, and then none for the rest of the follow-up period the same as those who have the same number of events evenly spread out throughout the period.

We have concentrated on the point estimates, with no detailed examination of the variances of these. Thus, more questions remain to be answered about meta-analysis of count data outcomes analysed using alternative methods. The impact of the trial analytical method on meta-analytic intervention effects, their standard errors and heterogeneity needs to be investigated. The impact is likely to vary by the chosen meta-analysis model (random effects versus fixed effect), so any investigation should examine both models. This simulation only examined data that were missing completely at random. This is overly simplistic, and research examining the impact of different missing data mechanisms and how these interact with the trial and meta-analysis methods would be valuable.

The focus of this paper is on RCTs, but these methods of analysis are used for other types of studies (non-randomised trials, observational studies), which may also be included in meta-analyses. For study types other than RCTs, it would be critical to examine the impact of covariates and missing data, in addition to the examination we have undertaken in this paper.

\section{Conclusions}

We have shown in this simulation study, that analysing outcomes using different methods yielded estimates of intervention effect that were similar in both average estimates and variances. When the mean of the counts is more than 0.5 , analyses using dichotomisation or time to the first event should not be pooled with intervention effects estimated from other methods. Dichotomising, when the event rate is at this level or higher, may not be an appropriate method for analysing individual studies as it is likely to underestimate treatment differences as well as giving confidence intervals that are too narrow.

\section{Additional file}

Additional file 1: The implications for meta-analysis of alternative methods for analysing count data. Additional file 1 contains three sections. The first is example Stata code for the simulations to make it absolutely clear how they were done. It is possible to present the results by using ratios of the results for the difference method rather than difference. These are presented in the second section. The third section contains the plots of the distributions of the differences for all the simulated scenarios. (DOCX 1,488 kb)

\section{Abbreviations}

Cl: confidence interval; HR: hazard ratio; RaR: rate ratio; RCT: randomised controlled trial; RR: risk ratio.

\section{Competing interests}

All authors declare that they have no conflicts of interest. CR was an investigator on two of the studies that provided data for the empirical study.

\section{Authors' contributions}

$\mathrm{PH}$ conceived of the idea in conjunction with $\mathrm{CR}$, carried out the simulations, wrote the first draft of the manuscript. CR helped with the original idea and revised the manuscript. JM refined the idea, helped with the simulations, and revised the manuscript. All authors read and approved the final manuscript.

\section{Acknowledgements}

This study was funded, in part, by a Health Research Council of New Zealand Grant (12-256). We are grateful to Emeritus Professor A. John Campbell for his valuable contribution to this paper and to the trial authors who provided falls data from their randomised controlled trials for these analyses.

\section{Author details}

'Department of Preventive and Social Medicine, Dunedin School of Medicine, University of Otago, PO Box 913, Dunedin 9054, New Zealand. ${ }^{2}$ Department of Medicine, Dunedin School of Medicine, University of Otago PO Box 913, Dunedin 9054, New Zealand. ${ }^{3}$ School of Public Health and Preventive Medicine, Monash University, The Alfred Centre, 99 Commercial Road, Melbourne, Victoria 3004, Australia.

Received: 6 July 2015 Accepted: 26 October 2015 Published online: 17 November 2015

\section{References}

1. Cameron AC, Trivedi PK. Regression analysis of count data. 2nd ed. Cambridge: Cambridge University Press; 2013.

2. Coxe S, West SG, Aiken LS. The analysis of count data: a gentle introduction to Poisson regression and its alternatives. J Pers Assess. 2009;91(2):121-36. doi:10.1080/00223890802634175. 
3. Donaldson MG, Sobolev B, Cook WL, Janssen PA, Khan KM. Analysis of recurrent events: a systematic review of randomised controlled trials of interventions to prevent falls. Age Ageing. 2009;38(2):151-5. doi:10.1093/ ageing/afn279.

4. Friedrich JO, Adhikari NK, Beyene J. The ratio of means method as an alternative to mean differences for analyzing continuous outcome variables in meta-analysis: a simulation study. BMC Med Res Methodol. 2008:8:32.

5. Gillespie LD, Robertson MC, Gillespie WJ, Lamb SE, Gates S, Cumming RG, Rowe $\mathrm{BH}$. Interventions for preventing falls in older people living in the community. Cochrane Database of Systematic Reviews 2009, Issue 2. Art. No. CD007146. doi:10.1002/14651858.CD007146.pub2.

6. Agresti A. 2012. Categorical data analysis, third edition. Wiley, doi:10.1111/ biom. 12128 .

7. Hilbe JM. Negative binomial regression. 2nd ed. Cambridge: Cambridge University Press; 2011.

8. Cox DR. Regression models and life-tables. Journal of the Royal Statistics Society, Series B. 1972;34(2):187-220.

9. Stürmer T, Glynn RJ, Kliebsch U, Brenner H. Analytic strategies for recurrent events in epidemiologic studies: background and application to hospitalization risk in the elderly. J Clin Epidemiol. 2000;53(1):57-64. doi:10.1016/S0895-4356(99)00137-7.

10. Andersen PK, Gill RD. Cox's regression model for counting processes: a large sample study. Ann Stat. 1982;10(4):1100-20.

11. Robertson MC, Campbell AJ, Herbison P. Statistical analysis of efficacy in falls prevention trials. Journals of Gerontology Series A-Biological Sciences \& Medical Sciences. 2005;60(4):530-4. doi:10.1093/gerona/60.4.530.

12. Guevara JP, Berlin JA, Wolf FM. Meta-analytic methods for pooling rates when follow-up duration varies: a case study. BMC Med Res Methodol. 2004:4:17. doi:10.1186/1471-2288-4-17.

13. Gardner W, Mulvey EP, Shaw EC. Regression analyses of counts and rates: Poisson, overdispersed Poisson, and negative binomial models. Psychol Bull. 1995;118(3):392-404. doi:10.1037/0033-2909.118.3.392.

14. Glynn RJ, Buring JE. Ways of measuring rates of recurrent events. BMJ. 1996;312(7027):364-7. doi:10.1136/bmj.312.7027.364.

15. Lord SR, Castell S, Corcoran J, Dayhew J, Matters B, Shan A, et al. The effect of group exercise on physical functioning and falls in frail older people living in retirement villages: a randomized, controlled trial. J Am Geriatr Soc. 2003;51(12):1685-92. doi:10.1046/j.1532-5415.2003.51551.x.

16. Harris RJ, Bradburn MJ, Deeks JJ, Harbord RM, Altman DG, Sterne JAC. Metan: fixed- and random-effects meta-analysis. Stata J. 2008;8(1):3-28

17. Mantel N, Haenszel W. Statistical aspects of the analysis of data from retrospective studies of disease. J Natl Cancer Inst. 1959;22:719-48. doi:10.1093/jnci/22.4.719.

18. DerSimonian R, Laird N. Meta-analysis in clinical trials. Control Clin Trials. 1986;7:177-88. doi:10.1016/0197-2456(86)90046-2.

19. Barnett A, Smith B, Lord SR, Williams M, Baumand A. Community-based group exercise improves balance and reduces falls in at-risk older people: a randomised controlled trial. Age Ageing. 2003;32(4):407-14. doi:10.1093/ ageing/32.4.407.

20. Campbell AJ, Robertson MC, Gardner MM, Norton RN, Tilyard MW, Buchner DM. Randomised controlled trial of a general practice programme of home based exercise to prevent falls in elderly women. BMJ. 1997;315(7115):1065-9. doi:10.1136/bmj.315.7115.1065

21. Campbell AJ, Robertson MC, Gardner MM, Norton RN, Buchner DM. Psychotropic medication withdrawal and a home-based exercise program to prevent falls: a randomized, controlled trial. J Am Geriatr Soc. 1999;47(7):850-3.

22. Green J, Forster A, Bogle S, Young J. Physiotherapy for patients with mobility problems more than 1 year after stroke: a randomised controlled trial. Lancet. 2002;359(9302):199-203. doi:10.1016/S0140-6736(02)07443-3.

23. Lord SR, Ward JA, Williams P, Strudwick M. The effect of a 12-month exercise trial on balance, strength, and falls in older women: a randomized controlled trial. J Am Geriatr Soc. 1995;43(11):1198-206.

24. Robertson MC, Devlin N, Gardner MM, Campbell AJ. Effectiveness and economic evaluation of a nurse delivered home exercise programme to prevent falls. 1: Randomised controlled trial. BMJ. 2001;322(7288):697-701. doi:10.1136/bmj.322.7288.697.

25. Skelton D, Dinan S, Campbell M, Rutherford O. Tailored group exercise (Falls Management Exercise-FaME) reduces falls in community-dwelling older frequent fallers (an RCT). Age Ageing. 2005;34(6):636-9. doi:10.1093/ageing/afi174
26. Slymen DJ, Ayala GX, Arredondo EM, Elder JP. A demonstration of modeling count data with an application to physical activity. Epidemiologic Perspectives \& Innovations. 2006;3:3. doi:10.1186/1742-5573-3-3.

27. Price RM, Bonett DG. Distribution free confidence intervals for difference and ratio of medians. J Stat Comput Simul. 2002;72(2):119-24. doi:10.1080/ 00949650212140.

28. Seeber GUH. Poisson regression. Encyclopedia of Biostatistics, Wiley. 2005. doi:10.1002/0470011815.b2a10044.

29. Aaron SD, Fergusson D, Marks GB, Suissa S, Vandemheen KL, Doucette $S$, et al. Counting, analysing and reporting exacerbations of COPD in randomised controlled trials. Thorax. 2008;63(2):122-8. doi:10.1136/ thx.2007.082636

\section{Submit your next manuscript to BioMed Central and take full advantage of:}

- Convenient online submission

- Thorough peer review

- No space constraints or color figure charges

- Immediate publication on acceptance

- Inclusion in PubMed, CAS, Scopus and Google Scholar

- Research which is freely available for redistribution

Submit your manuscript at www.biomedcentral.com/submit
C Biomed Central 